\title{
Mudança de Cultura no Uso de Tecnologias Educacionais: Estudo de Caso no Modelo Semipresencial do Cederj
}

\author{
Daniel Fábio Salvador*1, Luiz Gustavo Ribeiro Rolando², Clarisse de \\ Mendonça e Almeida ${ }^{3}$, Judith Almeida de Mello ${ }^{4}$
}

\footnotetext{
${ }^{1}$ Doutor/Professor/ Pesquisador, Fundação Centro de Ciências e Educação Superior a Distância, Rua da Ajuda, n 5. $15^{\circ}$ andar - Centro CEP: 20040-000 - RJ. salvador@cecierj.edu.br

2 Doutorando, Laboratório de Avaliação em Ensino e Filosofia das Biociências. Instituto Oswaldo Cruz. Av. Brasil, 4365 - Rio de Janeiro - RJ - Brasil. gustavor@ioc.fiocruz.br

3 Mestre, Fundação Cecierj, Fundação Centro de Ciências e Educação Superior a Distância, Rua da Ajuda, n 5. $15^{\circ}$ andar - Centro CEP: 20040-000 - RJ. cmalmeida@cecierj.edu.br

4 Graduada, Fundação Cecierj, Fundação Centro de Ciências e Educação Superior a Distância, Rua da Ajuda, n 5. $15^{\circ}$ andar - Centro CEP: 20040-000 - RJ. jmello@cecierj.edu.br
}

\section{Resumo}

O presente trabalho se propõe a descrever o processo de implementação de uma nova cultura de uso do ambiente virtual de aprendizagem, iniciado em 2012, junto aos cursos de graduação do Consórcio Cederj, ofertados no modelo semipresencial, e, posteriormente, sua difusão pelas diversas disciplinas. Com o objetivo principal de se aprimorar os processos de ensino-aprendizagem a distância, foram instituídas, além da implementação de uma nova plataforma, ações de capacitação destinadas aos diversos atores envolvidos no processo e iniciativas de apoio ao atendimento ao aluno e ao professor no que se refere à edição e ao uso de salas de aula virtuais. Dados coletados e apresentados ao longo do presente trabalho trazem reflexões relevantes acerca da utilização da plataforma de cursos on-line, em larga escala, baseada em uma abordagem construtivista e de acordo com os princípios atuais do design instrucional contextualizado e de estímulo à autoria do professor.

Palavras-chave: Educação a distância; Web 2.0; Sistema de gestão da aprendizagem. 


\title{
Culture Change in the Use of Educational Technology: a Case Study in Cederj Blended Model
}

\begin{abstract}
This paper aims to describe the implementation process of a new culture on using a virtual learning environment, which started in 2012, in undergraduate courses offered by Consórcio Cederj in a blended learning model, and, later, to discuss the propagation of this culture for all courses. With the objective of improving the distance teaching and learning process, a new learning platform was implemented. In addition training programs were instituted for the actors involved in the process and support initiatives to student and teacher in order to help them editing and using the virtual classrooms. Collected and presented data present in this work brings relevant ideas about the use of learning platform to offer online courses, in a large-scale, based on a constructivist approach and according to current principles of contextualized instructional design and of stimulus to the professor's authorship.
\end{abstract}

Key words: Distance education; Web 2.0; Learning management system. 


\section{Introdução}

Partindo de uma proposta de aprimoramento dos processos de ensinoaprendizagem suportados pelas ferramentas ofertadas pela Web 2.0 no que tange à Educação a Distância e baseados nos princípios norteadores da aprendizagem colaborativa apoiada por computadores, pelo designer instrucional contextualizado (Filatro, 2004) e pelo desenvolvimento da autonomia na autoria do professor de EaD na condução de sua sala de aula on-line, diversas ações foram realizadas durante o ano de 2012 no sentido de implementar uma nova cultura para o uso de uma sala de aula dinâmica e contextualizada voltada para os cursos de graduação a distância do Consórcio Cederj.

Levando-se em consideração uma comunidade de cerca de 30.000 usuários ativos - entre alunos, professores e corpo técnico -, acredita-se ser fundamental propor uma reflexão acerca desse processo de implementação e difusão de novas tecnologias para EaD, destacando sua aplicação em cursos a distância e a consolidação de uma Cultura Digital, em larga escala em docentes que, até então, trabalhavam somente com ensino presencial. Nesse sentido, o que se propõe é descrever as principais etapas desse processo destacando a atuação dos atores envolvidos, os resultados das ações de capacitação e de suporte e a quantificação do trabalho de edição realizado nas salas de aula virtuais ao longo desse período de transição.

\section{Fundamentação Teórica}

A adoção de novas habilidades e práticas didáticas por professores requer uma série contínua de esforços coordenados passando por oportunidades de desenvolvimento profissional vitalício (Bransford, Brown, \& Cocking, 2000; DarlingHammond \& Bransford, 2005), tornando-se, assim, um processo de atualização de suas bases de conhecimento e de apropriação de novas tecnologias. A troca de experiências e a contextualização do conhecimento compartilhado nas atividades de formação continuada devem estar relacionadas ao dia a dia do exercício profissional, em um contínuo processo de ação-reflexão-ação (Bonzanini \& Bastos, 2009; Schon, 1983). Nesse sentido, o desenvolvimento profissional dos professores é considerado componente crucial para a melhoria educacional (Brasil, 2006; CONAE, 2010; UNESCO, 2008). Entretanto, a prática docente é sabidamente marcada pela elevada carga 
horária, pela multiplicidade de vínculos empregatícios individuais que, em conjunto, contribuem para a sobrecarga profissional e para a redução das oportunidades de formação continuada (Ballet \& Kelchtermans, 2009).

No Brasil, a utilização de TICs e da internet, em especial na modalidade de Educação a Distância (EaD), tanto na graduação quanto na pós-graduação, cresceu exponencialmente na última década (INEP-MEC, 2010). Os dados oficiais demonstram que o percentual de alunos matriculados em cursos de graduação nessa modalidade subiu de 0,2\% em 2001 para 14,6\% do total de alunos em 2009, alcançando cerca de 800.000 pessoas naquele ano. O modelo brasileiro de promoção de Educação a Distância pública, patrocinado pela Universidade Aberta do Brasil, prevê que o professor do sistema presencial que queira aderir a essa modalidade de ensino, pode participar de programas de ensino semipresencial dessas universidades, recebendo adicional salarial em bolsas para conduzir suas disciplinas semipresenciais juntamente com o ensino presencial. Somada a isso, a legislação específica do MEC autorizou todas as universidades presenciais a oferecer até $20 \%$ dos seus conteúdos a distância desde que com as adequadas tecnologias. Isso possibilitou que professores de nível superior possam ministrar até $20 \%$ do conteúdo da sua disciplina ou $20 \%$ de disciplinas do seu curso de forma totalmente on-line através da modalidade à distância.

Todo esse contexto de introdução de novas tecnologias educacionais dentro das universidades brasileiras impacta seus modelos de ensino e a forma como esses professores precisam ser capacitados para lidar com inovações no sentido de que exigem novas bases de conhecimento e habilidades, antes não necessárias no modelo presencial. Apesar dessas diversas iniciativas de promoção de EaD e uso de tecnologias educativas pelo governo federal, o professor universitário brasileiro não integra tecnologia no seu ensino, sendo isso um entrave para a implementação de políticas públicas que almejam ampliar o acesso à educação por meio das TICs e EaD (CONAE, 2010), sendo bastante necessário ao crescimento do país, maior aprofundamento em pesquisas significativas e com resultados empíricos que direcionem ações nessa direção.

Falando-se em difusão e adoção de inovações tecnológicas, a primeira perspectiva teórica a ser colocada em qualquer trabalho de pesquisa é a teoria de difusão de inovação (Rogers, 2003). Além dessa teoria, existem diversos outros estudos na literatura internacional que fornecem aporte à pesquisa da área, baseados ou não nos 
fundamentos de Rogers, sendo os mais citados e usados no campo da tecnologia educacional: a teoria do aprendizado social (Bandura, 1977); o modelo CBAM (Concern-based Adoption model) (Hall \& Hord, 1987), bem como modelos relacionados especificamente ao processo de integração de TICs na educação, tais como: Technology Acceptance Model - TAM (Davis, 1989); Level of Technology Implementation- LoTI (Mehta \& Hull, 2012; Moersch, 1995); e o modelo de Delone e Mcclean para sucesso em sistemas de informação (DeLone \& Mclean, 2003; Lin, 2007). Além dos modelos citados, há ainda uma introdução ao framework teórico TPACK, que oferece um quadro de referência para a análise das práticas docentes com o uso de TICs (Mishra \& Koehler, 2006). Atualmente, o TPACK é um dos modelos teóricos mais pesquisados atualmente no campo de integração de tecnologias educacionais na prática docente(Chai et al., 2013; Graham, 2011; Voogt et al., 2013).

Todos os modelos teóricos de difusão e adoção de tecnologias podem ser utilizados em conjunto ou separadamente para servir como estrutura teórica que fundamentem a integração de tecnologias educacionais na prática docente. Um exemplo de aplicação desses fundamentos foi o programa de Faculty Technology Mentoring da Iowa State University (Thompson et al., 2007). O uso de Faculty Mentoring no processo de integração da tecnologia na prática docente é baseado no fato de que programas de formação continuada de professores, sem o suporte tecnológico individualizado, têm frequentemente falhado para atender às necessidades específicas da maioria dos professores (Sprague, Kopfman, \& Dorsey, 1998). Porém os programas de Faculty Technology Mentoring com atendimento individualizado (um a um) têm demonstrado resultados promissores na integração da tecnologia no currículo (Leh, 2005; Smith \& O’Bannon, 1999).

Especificamente no campo da educação a distância, Pratt \& Palloff (2011) citam a importância do processo de Faculty Mentoring para orientação de novos professores, em especial, a fim da implementação de um sistema de educação on-line (Palloff \& Pratt, 2011). Os autores citam revisão de literatura sobre a área (Chuang et al., 2003), destacando as abordagens e os elementos comuns aos principais programas de integração de tecnologias educacionais na prática docente, que vão desde a provisão da visão do uso de tecnologias para o aprendizado e ensino até a formação de comunidades de aprendizagem de professores em seus estágios mais avançados (Correia \& Davis, 2008). 


\section{Metodologia}

No ano de 2012, foi implementada a plataforma educacional Moodle/Cederj como ambiente virtual de aprendizagem voltado para as disciplinas de graduação ofertadas. Atrelado a isso, o Departamento de Mídias Digitais da Fundação Cecierj desenvolveu um conjunto de ações visando à mudança de cultura no uso dos ambientes virtuais de aprendizagem. As ações principais para estimular essa mudança de cultura e a difusão de novas tecnologias pelos coordenadores de disciplinas baseavam-se em:

- Implementação da plataforma educacional Moodle/Cederj, com visão socioconstrutivista possibilitando a autoria da sala de aula on-line pelo professor (coordenador de disciplina);

- Estabelecimento do atendimento individualizado para os coordenadores de disciplina com a introdução da figura do Tutor de Apoio ao Professor (TAP), fundamentado no modelo teórico Faculty Technology Mentory (Chuang, Thompson\& Schmidt, 2003);

- Criação de um setor de suporte especializado ao usuário voltado para a plataforma educacional Moodle/Cederj;

- Criação e divulgação de tutoriais para o uso das ferramentas e recursos;

- Oficinas presenciais e palestras de capacitação docente;

- Cursos on-line de capacitação docente visando à construção de AVAs, baseados nas melhores práticas de interação em EaD.

\section{Resultados e discussão}

Na Figura 1, é possível verificar o progresso na implementação e utilização dos ambientes virtuais de aprendizagem (AVA) criados pelos professores durante o ano 2012. Observa-se que, ao final do $1^{0}$ semestre, $75 \%$ das disciplinas possuíam pelo menos cinco seções editadas contra $35 \%$ no início desse mesmo ano. Ao final do ano de 2012, esse percentual já era de $83 \%$. Ambiente virtuais de aprendizagem baseados no paradigma da EaD apenas distributiva possuem geralmente diversos espaços para publicações de materiais didáticos em suas diferentes mídias, bem como ferramentas 
de interação com alunos, porém tendem a não possuir uma linha mestra de condução da aprendizagem dos alunos.

No caso da plataforma Moodle, as pastas de recursos, os documentos de orientação e as ferramentas de interação são preferencialmente dispostos em sua área central tecnicamente chamada de seções onde serão apresentadas as unidades de aprendizagem da disciplina. O fato de, no início do ano de 2012, já com a plataforma Moodle implementada, somente 35\% das disciplinas estarem usando essa área central, preferindo a disponibilização de matérias em pastas e links isolados, mostra que a maioria dos coordenadores de disciplina não tinha como paradigma a sala de aula virtual com local para condução cadenciada da aprendizagem dos estudantes. A evolução de $48 \%$ do início para o final do ano, provocada pelas diversas ações de difusão dessa cultura de uso mais intensivo do espaço virtual, é um claro indício que houve forte mudança da cultura de uso da plataforma de EaD no Consórcio Cederj.

Em relação ao uso de fóruns de discussão ativos, dentro das disciplinas no mês de fevereiro de 2012, havia apenas 39\% de disciplinas com essa ferramenta. Ao final do primeiro semestre, esse percentual subiu para $59 \%$ das disciplinas, fechando o segundo semestre letivo com 65\%. Para atividades on-line de envio de tarefa pela plataforma. esse aumento também foi notório de 34\% para $65 \%$ do início para o final do ano. O uso de fóruns e atividades de envio de tarefa dentro de um AVA são fortes indicativos cada vez mais desse ambiente como sala de aula interativa onde ações efetivas de colaboração aluno-aluno e aluno-tutores podem acontecer com maior frequência e qualidade.

O padrão recomendado para um AVA dentro do contexto do consórcio Cederj deveria possuir os seguintes itens básicos: cronograma e guia da disciplina, conter cinco ou mais seções editadas e utilizar fórum de discussão e atividade on-line. Ao considerar esses itens básicos, disseminados por meio das ações de mudança de cultura e da difusão de novas tecnologias, obteve-se um progresso geral de cerca de $40 \%$ de edição dos AVAs ao final de um ano de implementação.

A edição das unidades de aprendizagem dos AVAs (seções) era realizada pelos próprios professores na plataforma Moodle/Cederj, a partir de uma disciplina modelo bem como a inclusão de guia de disciplina e cronograma atualizado e o uso de ferramentas interativas como fórum de interação e atividade on-line, onde também é observado o aumento da utilização (Figura 1). 


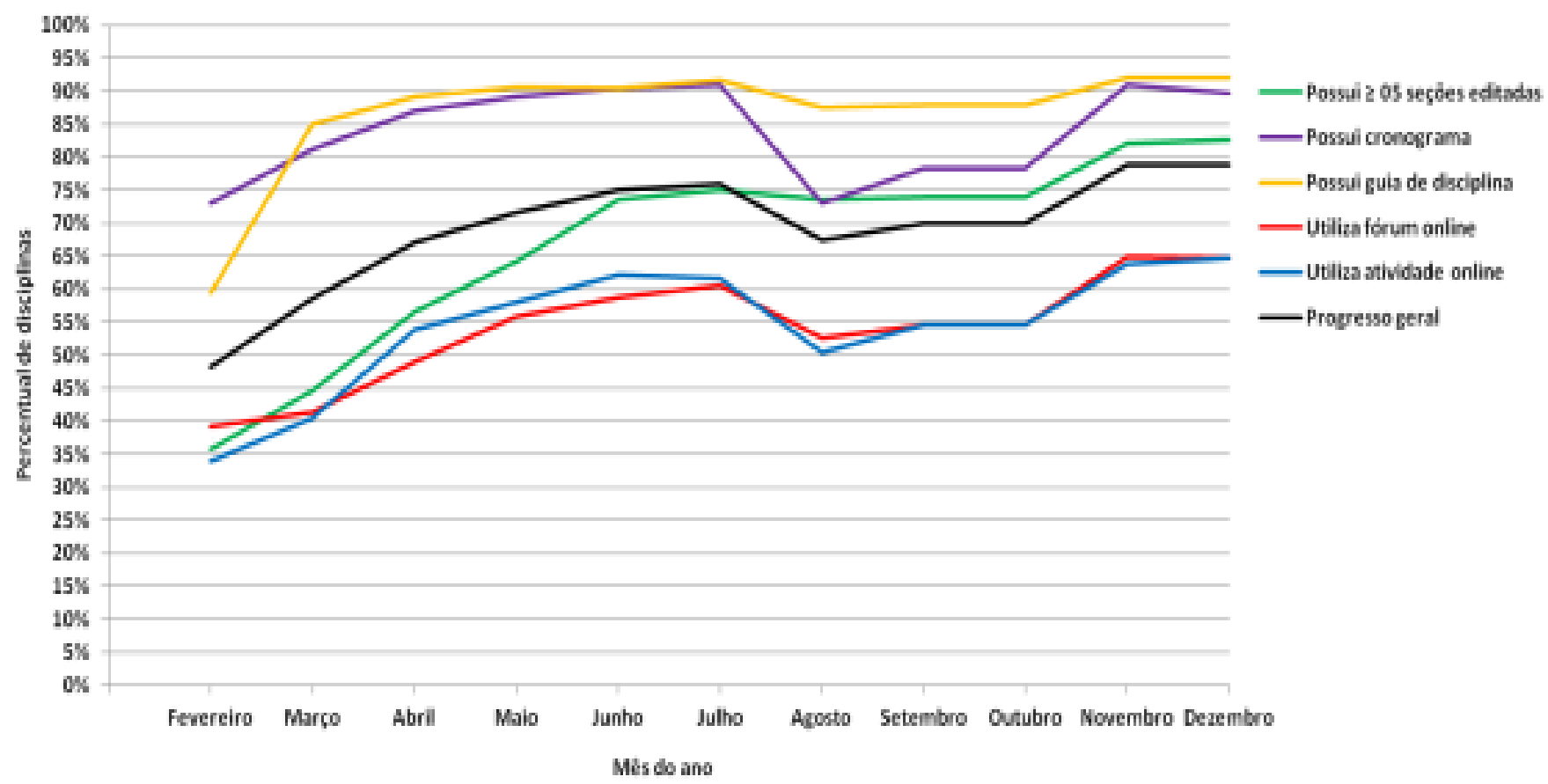

Figura 1: Progresso da edição das salas de aula on-line na plataforma Moodle CEDERJ durante $o$ ano de 2012 ( $n=536$ salas de aula on-line).

Diante do aumento dos índices de edição das salas de aula virtuais, definido como elemento central para indicar a mudança de cultura no uso da plataforma pelos professores, constata-se também o aumento da utilização da plataforma através dos logs de acesso dos usuários entre o ano de 2012 (ano de implementação da nova cultura) em comparação com o ano de 2013 (segundo ano do projeto). A figura 2, apresenta a comparação entre os meses do primeiro semestre para os anos de 2012 e 2013. Nota-se que houve aumento do total de atividades em todos os meses do semestre letivo, com destaque para os meses 3, 4 e 5, após a realização da Avaliação Presencial 1, indicando que, além do maior acesso no momento da avaliação presencial, a implementação de uma nova cultura ajudou na retenção da presença dos usuários da plataforma ao longo do semestre, resultando em maior presença virtual dos estudantes e professores do consórcio Cederj. 


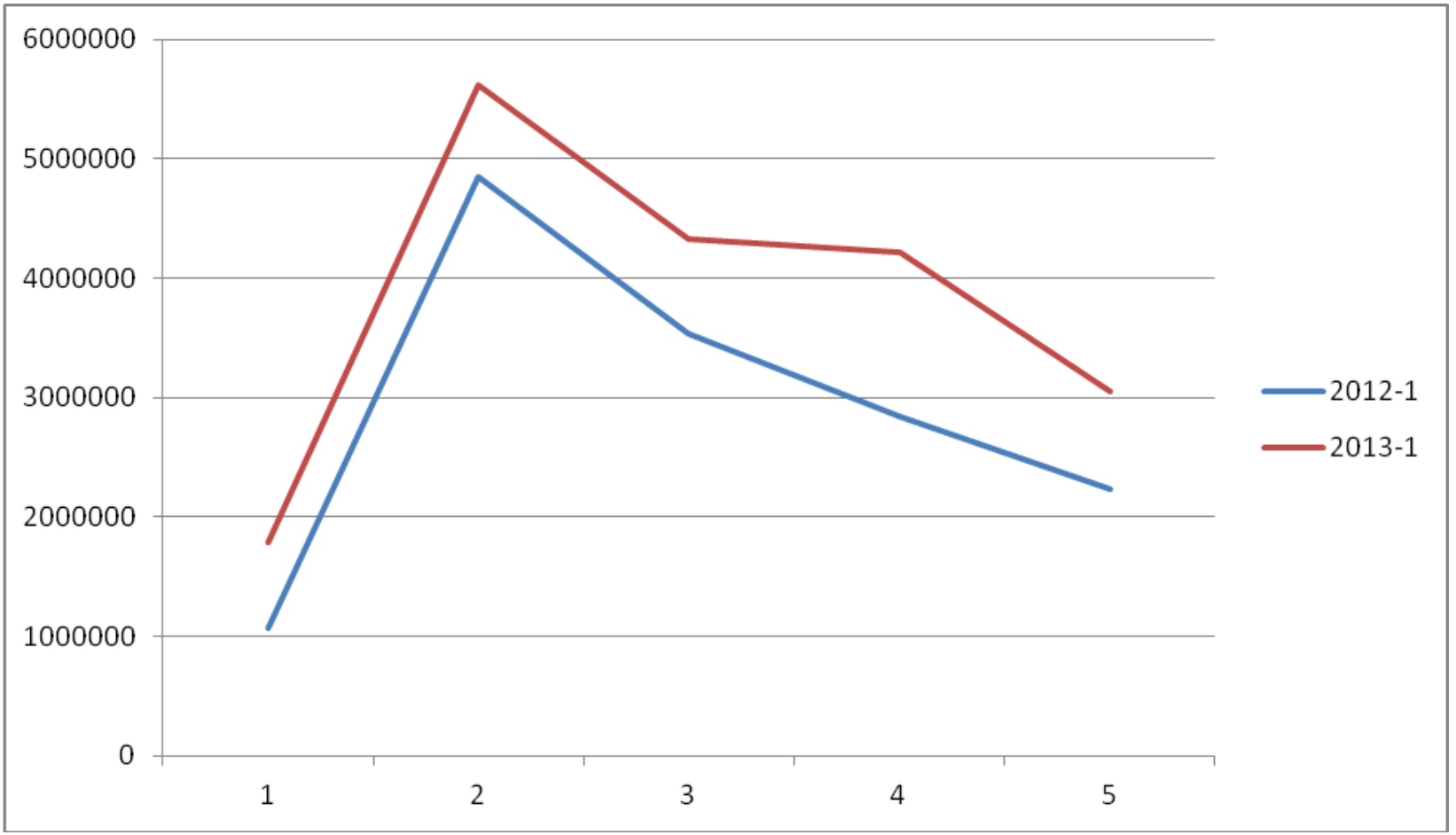

Figura 2:Total de atividades de todos os usuários no primeiro semestre (acesso a cada página interna das disciplinas).

\section{Relevância do resultado produzido e alcance nacional, regional ou local}

Nos anos iniciais do Consórcio Cederj, a produção de material multimídia estava diretamente atrelada ao material didático impresso, sendo esses dois elementos considerados os pilares centrais de qualidade desse sistema de ensino até o ano de 2010 juntamente com o forte apoio presencial ao aluno nos polos regionais. A plataforma, onde estavam disponibilizadas as salas de aulas virtuais, era utilizada apenas como um espaço de comunicação, e não de trocas, de interações e, consequentemente, de construção de conhecimento entre professores, tutores e alunos. A partir do ano de 2012, notou-se a necessidade de atualização desse modelo, levando todo o sistema a uma inversão de sua filosofia de EaD. A partir desse momento, os professores e tutores considerariam a sala de aula virtual como um espaço central da ocorrência de interações e colaboração (Van Bruggen, 2005) e não como ferramenta complementar. Essa percepção ocorreu pela massiva disseminação das novas tecnologias colaborativas da Web 2.0 (Brown, 2012; Sadaf, Newby \& Ertmer, 2012) bem como a evolução técnica de software para plataformas educacionais com foco na criação autoral do professor na web, como por exemplo, a 
plataforma Moodle. Este Sistema de Gerenciamento de Aprendizagem (LMS - Learning Management Systems), desenvolvido a partir de uma visão socioconstrutivista, foi implementado em larga escala no Consórcio Cederj para mais de 30 mil alunos, 2 mil tutores e 500 professores a partir do primeiro semestre de 2012.

Entretanto, somente a mudança do LMS usado no Consórcio Cederj não surtiria o efeito desejado, caso essa mudança não fosse acompanhada também de um programa de apoio docente que disseminasse os fundamentos e práticas eficazes de uso de tecnologias educacionais para educação on-line (Baran et al., 2011). O uso de Faculty Mentoring no processo de integração da tecnologia na prática docente é baseado no fato de que programas de formação continuada de professores, sem o suporte tecnológico individualizado, têm frequentemente falhado para atender às necessidades específicas da maioria dos professores (Sprague, Kopfman, \& Dorsey, 1998). Visando superar essa limitação, os programas de Faculty Technology Mentoring, com atendimento individualizado (um a um), têm demostrado resultados promissores na integração da tecnologia no currículo (Leh, 2005; Smith \& O’Bannon, 1999).

Especificamente no campo da educação a distância, Pratt \& Palloff (2011) citam a importância do processo de Faculty Mentoring para orientação de novos professores, em especial para implementação de sistema de educação on-line (Palloff \& Pratt, 2011). Os autores citam revisão de literatura sobre a área (Chuang et al., 2003), que destaca as abordagens e elementos comuns aos principais programas de integração de tecnologias educacionais na prática docente, indo desde a provisão da visão do uso de tecnologias para o aprendizado e ensino até a formação de comunidades de aprendizagem de professores em seus estágios mais avançados (Correia \& Davis, 2008).

\section{Nota}

1 O Consórcio Cederj é formado por sete instituições públicas de ensino superior: CEFET, UENF, UERJ, UFF, UFRJ, UFRRJ e UNIRIO, e conta atualmente com mais de 26 mil alunos matriculados em seus 12 cursos de graduação a distância. 


\section{Referências bibliográficas}

Ballet, K., \& Kelchtermans, G. (2009). Struggling with workload: Primary teachers' experience of intensification. Teaching and Teacher Education, 25(8), 11501157. Disponível em doi:10.1016/j.tate.2009.02.012

Bandura, A. (1977). Self-efficacy: toward a unifying theory of behavioral change. Psychological review, 84(2), 191-215. Disponível em doi:10.1037/0033$\underline{295 X .84 .2 .191}$

Baran, E., Correia, A.-P., \& Thompson, A. (2011). Transforming online teaching practice: critical analysis of the literature on the roles and competencies of online teachers. Distance Education, 32(3), 421-439. Disponível em doi: $10.1080 / 01587919.2011 .610293$

Bonzanini, T. K., \& Bastos, F. (2009). Formação continuada de professores de ciências: algumas reflexões. In Encontro Nacional De Pesquisa Em Educação Em Ciências (pp. 1 -12). Florianópolis: ABRAPEC.

Bransford, J. D., Brown, A. L., \& Cocking, R. R. (2000). How People Learn: Brain, Mind, Experience, and School (Expanded E., p. 384). Washington, D.C.: National research Council.

Brasil. (2006). Orientações Curriculares para o Ensino Médio: Ciências da Natureza, matemática e suas tecnologias. Secretaria de Educação Média e Tecnológica/MEC. (Vol. 2, p. 140). Brasília, DF. Disponível em doi:ISBN 8598171-43-3

Brown, S. a. (2012). Seeing Web 2.0 in context: A study of academic perceptions. The Internet and Higher Education, 15(1), 50-57. Disponível em doi: 10.1016/j.iheduc.2011.04.003

Chai, C. S., Koh, J. H. L., \& Tsai, C. (2013). A Review of Technological Pedagogical Content Knowledge. Educational Technology \& Society, 16, 31-51.

Chuang, H., Thompson, A., \& Schmidt, D. (2003). Faculty Technology Mentoring Programs: Major Trends in the Literature. Journal of Computing in Teacher Education, 19(4), 101-106.

CONAE. (2010). Construindo o Sistema Nacional Articulado de Educação: o Plano Nacional de Educação, diretrizes e estratégias; documento Final. Brasília: INEP (pp. 1-168). Brasília, DF.

Correia, A., \& Davis, N. (2008). Intersecting communities of practice in distance education: the program team and the online course community. Distance Education, 29(3), 289-306. Disponível em doi:10.1080/01587910802395813

Darling-Hammond, L., \& Bransford, J. (2005). Preparing teachers for a changing world: What teachers should learn and be able to do. (L. Darling-Hammond \& J. Bransford, Eds.) (p. 583). San Francisco, CA: Jossey-Bass Inc. 
Davis, F., Bagozzi, R., \& Warshaw, P. (1989). User acceptance of computer technology: A comparison of two theoretical models. Management Science, 35, 982-1003.

DeLone, W., \& Mclean, E. R. (2003). The DeLone and McLean Model of Information Systems Success: A Ten-Year Update. Journal of Management information systems, 19, 9-31.

Filatro, A. (2004). Design instrucional contextualizado. Senac. São Paulo.

Graham, C. R. (2011). Theoretical considerations for understanding technological pedagogical content knowledge (TPACK). Computers \& Education. Disponível em doi: 10.1016/j.compedu.2011.04.010

Hall, G. E., \& Hord, S. M. (1987). Change in schools: facilitating the process. (p. 1987). Albaby, NY: New York State University Press.

INEP-MEC. (2010). Resumo Técnico: censo da educação superior de 2009 (pp. 01 37). Brasília, DF.

Leh, S. C. (2005). Lesson learned from service learning and reverse mentoring in Faculty development: A case study in technology training. Journal of technology and teacher education, 13(1), 25-41.

Lin, H.-F. (2007). Measuring online learning systems success: applying the updated DeLone and McLean model. Cyberpsychology \& behavior, 10(6), 817-20. Disponível em doi: $10.1089 /$ cpb.2007.9948

Mehta, V., \& Hull, D. M. (2012). Structural Validity of the Professional Development Profile of the LoTi Digital-Age Survey. Journal of Psychoeducational Assessment, 31(1), 61-71. Disponível em doi:10.1177/0734282912454992

Mishra, P., \& Koehler, M. J. (2006). Technological Pedagogical Content Knowledge: A Framework for Teacher Knowledge. (T. Bastiaens, J. Dron, \& C. Xin, Eds.)Teachers College Record, 108(6), 1017-1054. Disponível em doi: $10.1111 / j .1467-9620.2006 .00684 . x$

Moersch, C. (1995). Levels of technology implementation (LoTi): A frame- work for measuring classroom technology use. Learning and Leading with Technology, 23(3), 40-42., 23(3), 40-42.

Palloff, R. M., \& Pratt, K. (2011). The Excellent Online Instructor: Strategies for Professional Development (p. 176). San Francisco: Jossey-Bass.

Rogers, E. M. (2003). Diffusion of Innovations, 5th Edition. Book (p. 512).

Sadaf, A., Newby, T. J., \& Ertmer, P. a. (2012). Exploring pre-service teachers' beliefs about using Web 2.0 technologies in $\mathrm{K}-12$ classroom. Computers \& Education, 59(3), 937-945. Disponível em doi:10.1016/j.compedu.2012.04.001

Schon, D. A. (1983). The Reflective Practitioner: How Professionals Think In Action ( $p$. 365). New York: Basic Books. 
Smith, S. J., \& O'Bannon, B. (1999). Faculty Members infusing technology across teacher education: A mentorship model. Teacher Education and Special Education, 22(2), 123-135.

Sprague, D., Kopfman, K., \& Dorsey, S. (1998). Faculty development in the integration of technology in teacher edcucation courses. Journal of Computing in Teacher Education, 14(2), 24-28.

Thompson, A. D., Chuang, H., \& Sahin, I. (2007). Faculty Mentoring: The Power of Students in Developing Technology Expertise (p. 162). Charlote, US: Information age publishing.

UNESCO. (2008). ICT competency standards for teachers (p. 15). Paris, France.

Van Bruggen, J. (2005). Theory and practice of online learning. (T. Anderson \& F. Elloumi, Eds.) British Journal of Educational Technology, 36(1), 111-112. Disponível em: Disponível em doi:10.1111/j.1467-8535.2005.00445 1.x

Voogt, J., Fisser, P., Pareja Roblin, N., Tondeur, J., \& van Braak, J. (2013). Technological pedagogical content knowledge - a review of the literature. Journal of Computer Assisted Learning, 29(2), 109-121. Disponível em doi: 10.1111/j.1365-2729.2012.00487.x 